\title{
Effects of the $\mathrm{H}_{2} \mathrm{~S}$-donor AP39 during murine haemorrhage and resuscitation
}

\author{
Weidgang $\mathrm{C}^{1,2}$, Huber-Lang $\mathrm{M}^{3}$, Wood $\mathrm{M}^{4}$, Whiteman $\mathrm{M}^{4}$, Radermacher $\mathrm{P}^{1,2}$, Gröger $\mathrm{M}^{1}$ \\ Nußbaum $B^{1,2}$ \\ ${ }^{1}$ Institute of Anaesthesiological Pathophysiology and Process Development, University Medical School, Ulm, Germany \\ ${ }^{2}$ Department of Anaesthesiology, University Hospital, Ulm, Germany \\ ${ }^{3}$ Department of Traumatology, Hand-, Plastic- and Reconstructive Surgery, University Hospital, Ulm, Germany \\ ${ }^{4}$ University of Exeter Medical School, St. Luke's Campus, Exeter, UK
}

Background: The mitochondria-targeted hydrogen sulphide $\left(\mathrm{H}_{2} \mathrm{~S}\right)$-donor AP39 has previously been shown to exert cytoprotective effects in vitro by stimulation of mitochondrial respiration [1]. In vivo, AP39 improved haemodynamic and neurological outcome following cardiac arrest after pre- or early post-treatment administration [2,3]. Therefore, we tested the hypothesis whether AP39 has a beneficial impact in a resuscitated murine model of haemorrhagic shock.

Methods and measurements: Anaesthetised and instrumented mice (C57BL/6J) underwent $1 \mathrm{~h}$ of haemorrhage (mean arterial pressure (MAP)= $35 \mathrm{mmHg}$ ) and subsequently $4 \mathrm{~h}$ of resuscitation comprising a bolus injection of $100 \mathrm{nmol} / \mathrm{kg}$ AP39 $(n=8)$ or vehicle $(n=9)$, re-transfusion of shed blood together with lung-protective mechanical ventilation, fluid resuscitation and noradrenaline (NA) titrated to maintain MAP $>50 \mathrm{mmHg}$. Lung mechanics and gas exchange were assessed together with systemic haemodynamics, metabolism, and acid-base status. Blood and tissue samples were analysed for cytokine and chemokine levels, protein expression and high resolution respirometry to assess tissue mitochondrial activity (OxPHOS: maximal oxidative phosphorylation; LEAK: strong limitation of mitochondrial respiration due to ATP-synthase inhibition; ETS: maximal $\mathrm{O}_{2}$ consumption in the uncoupled state). Data are median (quartiles).
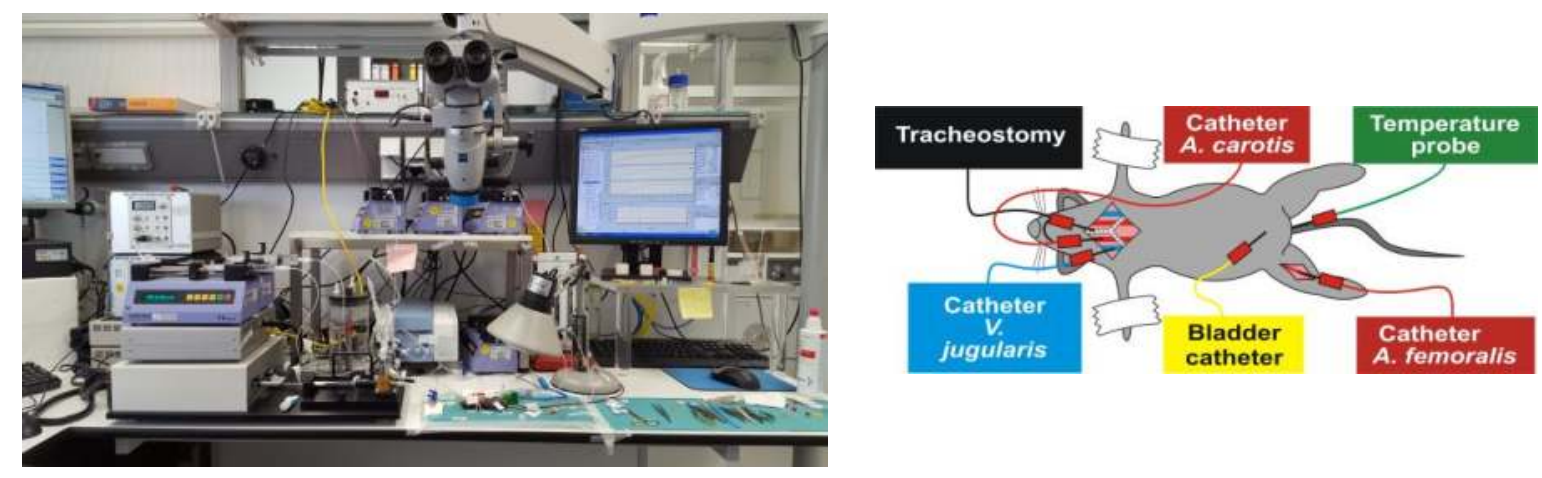

Results: AP39-treated mice revealed higher NA requirements to maintain haemodynamic stability which coincided with more pronounced metabolic acidosis and increased mortality rates (Table 1; Figure 1). Local and systemic inflammation was decreased following AP39 treatment (Table 2). In line, protein levels of the nitrosative stress marker (iNOS) were reduced in lung tissue whereas anti-inflammatory expression levels of HO-1 were increased (Figure 2). Figure 3 shows no significant effect of AP39 on OxPHOS, LEAK or ETS in diaphragm, heart, kidney and liver.
Table 1

\begin{tabular}{|c|c|c|c|}
\hline \multirow{2}{*}{ Parameters } & \multicolumn{2}{|c|}{ Haemorrhagic shock } & \multirow{2}{*}{$\begin{array}{c}\text { p-value } \\
\text { MWW-test }\end{array}$} \\
\hline & Vehicle & AP39 & \\
\hline Heart rate $\left[\mathrm{min}^{-1}\right]$ & $370(342 ; 515)$ & $380(325 ; 520)$ & 0.9156 \\
\hline MAP $[\mathrm{mmHg}]$ & $59(53 ; 64)$ & $50(43 ; 57)$ & 0.0796 \\
\hline Static compliance $\left[\mathrm{mL} \cdot \mathrm{cmH}_{2} \mathrm{O}^{-1}\right]$ & $0.12(0.11 ; 0.14)$ & $0.12(0.11 ; 0.13)$ & 0.7911 \\
\hline Noradrenaline $\left[\mu \mathrm{g} \cdot \mathrm{kg}^{-1} \cdot \mathrm{min}^{-1}\right]$ & $0.01(0.00 ; 0.02)$ & $0.07(0.02 ; 0.29) \#$ & 0.0261 \\
\hline $\mathrm{PaO}_{2} / \mathrm{FiO}_{2}[\mathrm{mmHg}]$ & $381(360 ; 457)$ & $371(313 ; 473)$ & 0.7282 \\
\hline Glucose $\left[\mathrm{mmol} \cdot \mathrm{L}^{-1}\right]$ & $5.1(3.7 ; 5.3)$ & $4.7(3.9 ; 5.5)$ & 0.8253 \\
\hline Arterial $\mathrm{pH}$ & $7.33(7.28 ; 7.37)$ & $7.27(7.18 ; 7.31) \#$ & 0.0401 \\
\hline Arterial $\mathrm{BE}\left[\mathrm{mmol} \cdot \mathrm{L}^{-1}\right]$ & $-5.6(-7.0 ;-3.1)$ & $-9.7(-14.8 ;-5.9)$ & 0.0541 \\
\hline Lactate $\left[\mathrm{mmol} \cdot \mathrm{L}^{-1}\right]$ & $0.9(0.8 ; 1.6)$ & $2.2(1.5 ; 3.8) \#$ & 0.0466 \\
\hline
\end{tabular}

Table 2

\begin{tabular}{|c|c|c|c|}
\hline \multirow{2}{*}{ Parameters } & \multicolumn{2}{|c|}{ Haemorrhagic shock } & \multirow{2}{*}{$\begin{array}{c}\text { p-value } \\
\text { MWW-test }\end{array}$} \\
\cline { 2 - 3 } & Vehicle & AP39 & \\
\hline KC $\left[\mathrm{pg} \cdot \mathrm{mL}^{-1}\right]$ & $314(251 ; 512)$ & $118(69 ; 284) \#$ & $\mathbf{0 . 0 0 2 0}$ \\
\hline $\mathrm{IL}-1 \beta\left[\mathrm{pg} \cdot \mathrm{mL}^{-1}\right]$ & $25(20 ; 28)$ & $24(18 ; 32)$ & $\mathbf{0 . 7 7 6 9}$ \\
\hline $\mathrm{IL}-6\left[\mathrm{pg} \cdot \mathrm{mL}^{-1}\right]$ & $275(144 ; 345)$ & $162(89 ; 340)$ & $\mathbf{0 . 1 1 9 0}$ \\
\hline $\mathrm{IL}-10\left[\mathrm{pg} \cdot \mathrm{mL}^{-1}\right]$ & $37(30 ; 42)$ & $27(14 ; 38)$ & $\mathbf{0 . 0 5 5 5}$ \\
\hline MCP-1 $\left[\mathrm{pg} \cdot \mathrm{mL}^{-1}\right]$ & $708(401 ; 1723)$ & $141(95 ; 365) \#$ & $\mathbf{0 . 0 0 0 1}$ \\
\hline TNF $\alpha\left[\mathrm{pg} \cdot \mathrm{mL}^{-1}\right]$ & $15(14 ; 23)$ & $10(7 ; 18) \#$ & $\mathbf{0 . 0 1 1 5}$ \\
\hline \# significant to Vehicle (p<0.05)
\end{tabular}

Figure 1

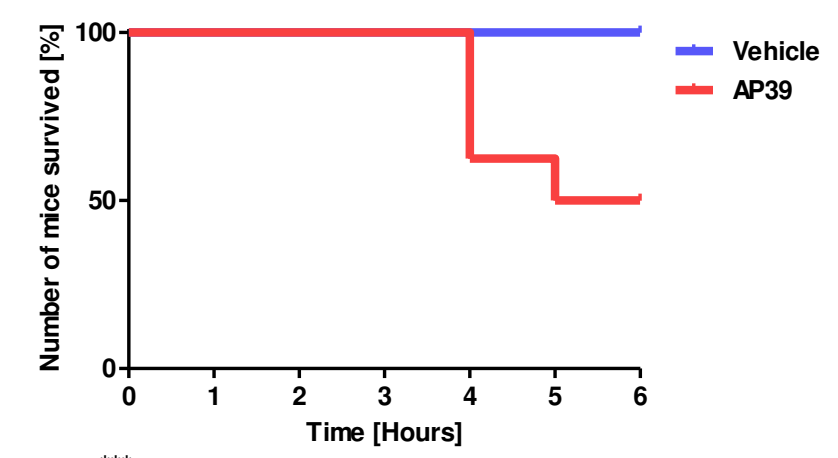

Figure 2
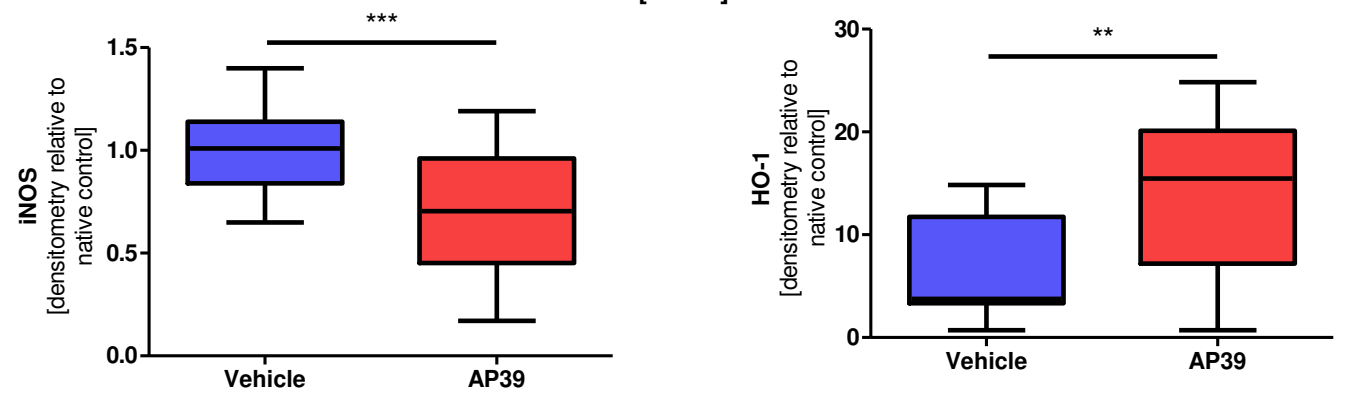

Figure 3
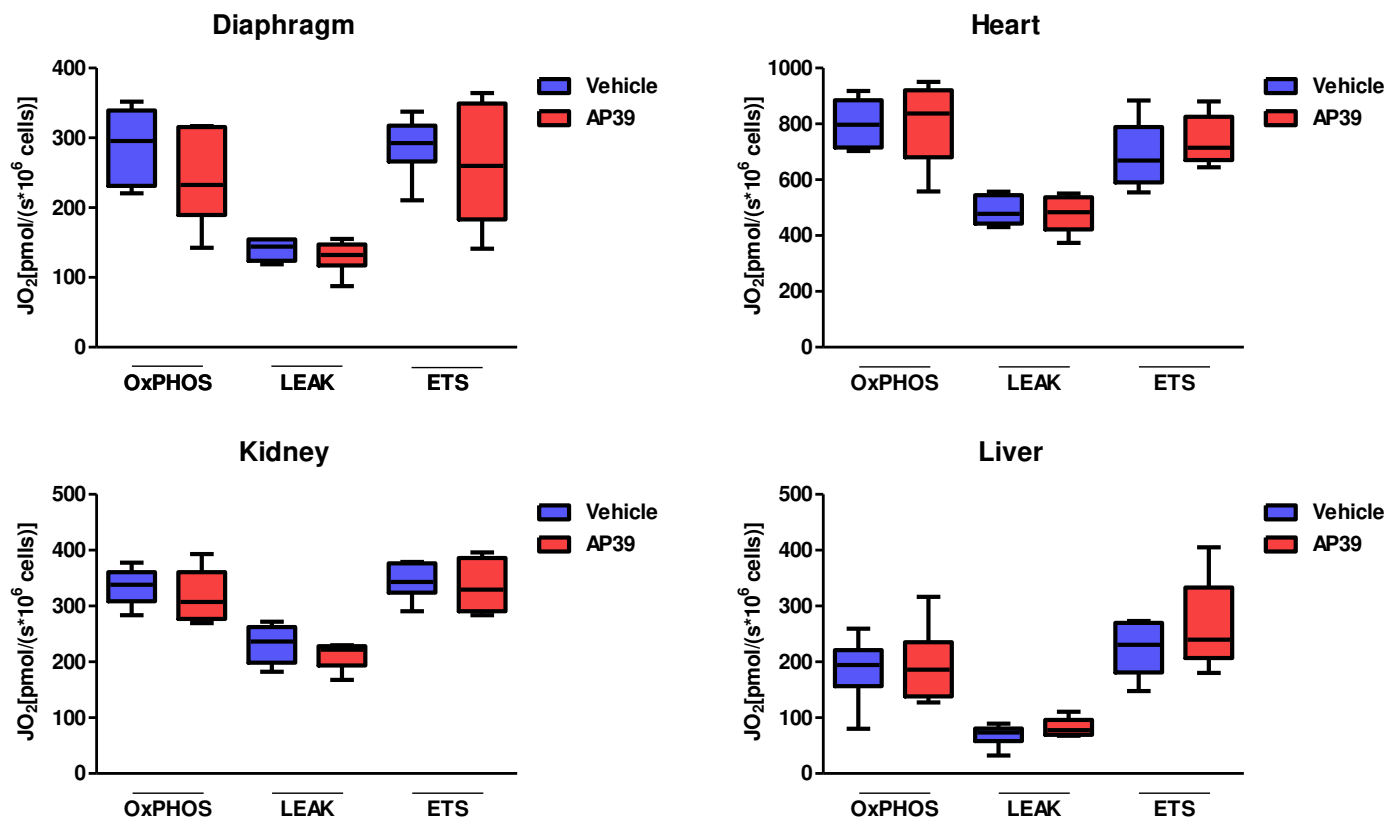

Conclusion: We were able to confirm the anti-inflammatory impact of AP39 in vivo. However, in contrast to the promising in vitro and in vivo data in cardiac arrest, AP39 had no beneficial effect on the outcome after murine haemorrhagic shock, possibly due to its timing and dose. In addition, the higher NA requirements, which may have resulted from AP39related vasodilation [3], might have further offset beneficial effects on mitochondrial respiration. 